\title{
ON THE RUIN PROBABILITY UNDER A CLASS OF RISK PROCESSES ${ }^{1}$
}

BY

\author{
WANG RONGMING AND LIU HAIFENG
}

\begin{abstract}
In this paper a class of risk processes in which claims occur as a renewal process is studied. A clear expression for Laplace transform of the finite time ruin probability is well given when the claim amount distribution is a mixed exponential. As its consequence, a well-known result about ultimate ruin probability in the classical risk model is obtained.
\end{abstract}

\section{KEYWORDS}

Sparre Andersen risk model, Finite time ruin probability, Laplace transform.

\section{INTRODUCTION}

Much of the literature on ruin theory is concerned with deriving results for the classical risk model, in which claims occur as a Poisson process. Sparre Andersen (1957) considered the situation in which claims occur as a general renewal process, and an explicit result for the ultimate ruin probability was derived for a particular case. Since then, much of the study of these models has concentrated on numerical procedures for calculating ruin probabilities [see, for example, Dickson (1998)]. Apart from purely mathematical breakthrough, Andersen's contribution allowed us to assume contagion between claims, i.e., to deal with non-Poissonian claims' arrivals. In fact, renewal nonPoissonian risk models do not look like a mere analytical over-complication, modern mass media and telecommunication networks could introduce substantial and sometimes unpredictable dependence into behaviour of insured persons which eventually could make an assumption on the Poissonian origin of claims' arrival suspicious [see Malinovskii (1998)].

In this paper a class of collective risk model with non-Poissonian claims' arrival processes is considered. A clear expression for Laplace transform of the finite time ruin probability is well given when the claim amount distribution

1 The work was partially supported by Fudan-Swiss Reinsurance Research Foundation (2001.6-2002.6). 
is the mixture of two exponentials. As its consequence, one result by Malinovskii (1998) about the expression for Laplace transform of the ruin probability within finite time is obtained when the claim amount distribution is a exponential. Finally, a well-known result in Gerber (1979) about ultimate ruin probability in the classical risk model is proved again.

This paper is organized as follows. Section 2 begins with some definitions, notations, and gives a main theorem and its proof. Two corollaries are given in Section 3.

\section{Main Result and Its Proof}

Consider a Sparre Andersen risk process

$$
R(t)=u+c t-\sum_{i=1}^{N(t)} Y_{i},
$$

defined in terms of the following values: $u=R(0) \geq 0$ is the initial risk reserve, $c>0$ is the premium received continuously per unit time, $\left\{T_{i}, i \geq 1\right\}$ are the (iid) interclaim time, $N(t)$ denotes number of claims having occurred up to time $t$, i.e., $N(t)=\max \left\{n: T_{1}+T_{2}+\cdots+T_{n} \leq t\right\}$, and $\left\{Y_{i}, i \geq 1\right\}$ are the (iid) amounts of claims. Throughout this paper, we suppose that $\left\{T_{i}, i \geq 1\right\}$ and $\left\{Y_{j}, j \geq 1\right\}$ are independent, and the relative security loading $\Lambda=\frac{c \cdot E T_{1}}{E Y_{1}}-1>0$, which means that the premium received per unit time exceed the expected claim payments per unit time. Denote by $\psi(t, u), \psi(u)$ and $\varphi(t, u)$ the probability of ruin within finite time, the probability of ultimate ruin and the probability of survival to time $t$, respectively. Clearly, $\psi(t, u)=1-\varphi(t, u)$. Our main result is the following theorem.

Theorem 1 Let the claim sizes $\left\{Y_{i}, i \geq 1\right\}$ and interoccurrence times $\left\{T_{i}, i \geq 1\right\}$ be mutually independent and i.i.d. Let $Y_{i}$ be a mixed exponential and its p.d.f. be $p \lambda_{1} e^{-\lambda_{1} y}+q \lambda_{2} e^{-\lambda_{2} y}, y>0,0<\lambda_{1}<\lambda_{2}, p+q=1,0 \leq p, q \leq 1$. Assume that $\gamma(a)$ $=\int_{0}^{\infty} e^{-a u} P_{T}(d u)$ is the Laplace transform of $T_{1}$, where $P_{T}(u)$ is the distribution function of $T_{1}$. Then

$$
a \int_{0}^{\infty} e^{-a t} \psi(t, u) d t=\frac{\left(\lambda_{1}-\lambda_{2}\right)\left[y_{1}(a) \beta_{2}(a) e^{-\beta_{1}(a) u}-y_{2}(a) \beta_{1}(a) e^{-\beta_{2}(a) u}\right]}{\left(\lambda_{1}-\beta_{2}(a)\right)\left(\lambda_{2}-\beta_{1}(a)\right)-\left(\lambda_{1}-\beta_{1}(a)\right)\left(\lambda_{2}-\beta_{2}(a)\right)}, a>0,
$$

where $y_{1}(a)=\left(1-\frac{\beta_{1}(a)}{\lambda_{1}}\right)\left(1-\frac{\beta_{1}(a)}{\lambda_{2}}\right), y_{2}(a)=\left(1-\frac{\beta_{2}(a)}{\lambda_{1}}\right)\left(1-\frac{\beta_{2}(a)}{\lambda_{2}}\right), \beta_{1}(a), \beta_{2}(a)$ are, respectively, the unique solutions of the equation

$$
\left(\lambda_{1}-\beta\right)\left(\lambda_{2}-\beta\right)-\left[\lambda_{1} \lambda_{2}-\left(p \lambda_{1}+q \lambda_{2}\right) \beta\right] \gamma(\alpha+c \beta)=0, \alpha>0
$$

in $\left(0, \lambda_{1}\right]$ and $\left[\beta_{0}, \lambda_{2}\right]$, and $\beta_{0}=\frac{\lambda_{1} \lambda_{2}}{p \lambda_{1}+q \lambda_{2}}$. 
Remark 1 Equation (2.2) has the unique root, respectively, in $\left(0, \lambda_{1}\right]$ and $\left[\beta_{0}, \lambda_{2}\right]$. In fact, Let

$$
f(\beta)=\left(\lambda_{1}-\beta\right)\left(\lambda_{2}-\beta\right)-\left[\lambda_{1} \lambda_{2}-\left(p \lambda_{1}+q \lambda_{2}\right) \beta\right] \gamma(\alpha+c \beta),
$$

then $f(0)>0, f\left(\lambda_{1}\right) \leq 0, f\left(\beta_{0}\right) \leq 0, f\left(\lambda_{2}\right) \geq 0$. By the existence theorem of root we know that Equation (2.2) has the roots in $\left(0, \lambda_{1}\right]$ and $\left[\beta_{0}, \lambda_{2}\right]$. On the other hand, it is very easy to prove the uniqueness of roots by the positiveness of relative security loading and the convexity of $\gamma(\alpha+c \beta)$ with respect to $\beta$.

Proof Let $\tau=\inf \{t>0: R(t)<0\}$ be the first time of ruin with the understanding that $\tau=\infty$ if $R(t) \geq 0$ for all $t$. Let $U_{n}=\sum_{i=1}^{n} T_{i}, U_{0}=0, v=\inf \{n \geq$ $\left.1: R\left(U_{n}\right)<0\right\}$, and $v$ is the index of that claim which causes the first ruin. If for each $n, R\left(U_{n}\right) \geq 0$, then $v=\infty$. Clearly, $\tau=U_{v}$. For $t, x \geq 0$, denote $Q^{n}(t, x)$ $=P\left\{U_{n} \leq t, R\left(U_{n}\right) \leq x, n<v\right\}, H(t, x)=\sum_{n \geq 0} Q^{n}(t, x)$, and Laplace transform of $H(t, x)$ denoted by $\hat{H}(a, \beta)$. Then

$$
\hat{H}(a, \beta)=\int_{0}^{\infty} \int_{0}^{\infty} e^{-a t-\beta x} H(d t, d x)=\sum_{n \geq 0} \mathrm{E}\left[e^{-a U_{n}-\beta R\left(U_{n}\right)} I_{\{v>n\}}\right], a, \beta>0 .
$$

Obviously, (2.3) is analytical. Since

$$
\begin{aligned}
\varphi(t, u) & =P(\tau>t) \\
& =\sum_{n \geq 0} P\left(\tau>t, U_{n} \leq t<U_{n+1}\right) \\
& =\sum_{n \geq 0} P\left(v>n, U_{n} \leq t, T_{n+1}>t-U_{n}\right) \\
& =\sum_{n \geq 0} \int_{0}^{t} \int_{0}^{\infty} Q^{n}(d \mu, d z)\left(1-P_{T}(t-\mu)\right) \\
& =\int_{0}^{t} H(d \mu, \infty)\left(1-P_{T}(t-\mu)\right),
\end{aligned}
$$

then we get

$$
d \varphi(t, u)=-\int_{0}^{t} P_{T}^{\prime}(t-\mu) H(d \mu, \infty) d t+H(d t, \infty)
$$

Consequently,

$$
\begin{aligned}
\alpha \int_{0}^{\infty} e^{-a t} \varphi(t, u) d t & =-\int_{0}^{\infty} \varphi(t, u) d e^{-a t} \\
& =\int_{0}^{\infty} e^{-a t} d \varphi(t, u) \\
& =\int_{0}^{\infty} \int_{0}^{t} e^{-a t}\left(-P_{T}^{\prime}(t-\mu)\right) H(d \mu, \infty) d t+\int_{0}^{\infty} e^{-a t} H(d t, \infty)
\end{aligned}
$$




$$
\begin{aligned}
& =\int_{0}^{\infty} \int_{0}^{t} e^{-a \mu} e^{-a(t-\mu)}\left(-P_{T}^{\prime}(t-\mu)\right) H(d \mu, \infty) d t+\hat{H}(a, 0) \\
& =\int_{0}^{\infty} e^{-a \mu}\left(\int_{\mu}^{\infty} e^{-a(t-\mu)}\left(-P_{T}^{\prime}(t-\mu)\right) d t\right) H(d \mu, \infty)+\hat{H}(a, 0) \\
& =\int_{0}^{\infty} e^{-a \mu} \int_{0}^{\infty} e^{-a y}\left(-P_{T}^{\prime}(y)\right) d y H(d \mu, \infty)+\hat{H}(a, 0) \\
& =-\int_{0}^{\infty} \gamma(a) e^{-a \mu} H(d \mu, \infty)+\hat{H}(a, 0) \\
& =\hat{H}(a, 0)(1-\gamma(a)) .
\end{aligned}
$$

Now we turn our attention to computation of $\hat{H}(a, 0)$.

To this end, denote $\Omega_{n}=\sigma\left\{Y_{1}, T_{1}, Y_{2}, T_{2}, \cdots, Y_{n}, T_{n}\right\}$. Noting that

$$
\begin{aligned}
& E\left[e^{-\beta R\left(U_{n}\right)} I_{\left\{R\left(U_{n}\right) \geq 0\right\}} \mid \Omega_{n-1}, T_{n}\right] \\
& =\int_{0}^{R\left(U_{n-1}\right)+c T_{n}} \exp \left\{-\beta\left(R\left(U_{n-1}\right)+c T_{n}-y\right)\right\}\left(p \lambda_{1} e^{-\lambda_{1} y}+q \lambda_{2} e^{-\lambda_{2} y}\right) d y \\
& =\frac{p \lambda_{1}}{\lambda_{1}-\beta}\left[e^{-\beta\left(R\left(U_{n-1}\right)+c T_{n}\right)}-e^{-\lambda_{1}\left(R\left(U_{n-1}\right)+c T_{n}\right)}\right] \\
& +\frac{q \lambda_{2}}{\lambda_{2}-\beta}\left[e^{-\beta\left(R\left(U_{n-1}\right)+c T_{n}\right)}-e^{-\lambda_{2}\left(R\left(U_{n-1}\right)+c T_{n}\right)}\right]
\end{aligned}
$$

we get

$$
\begin{aligned}
& E\left[e^{-a U_{n}-\beta R\left(U_{n}\right)} I_{\left\{R\left(U_{n}\right) \geq 0\right\}} \mid \Omega_{n-1}\right] \\
& =E\left[E\left(e^{-a U_{n}-\beta R\left(U_{n}\right)} I_{\left\{R\left(U_{n}\right) \geq 0\right\}}\left|\Omega_{n-1}, T_{n}\right| \Omega_{n-1}\right]\right. \\
& =E\left[e ^ { - a U _ { n } } \left(\frac{p \lambda_{1}}{\lambda_{1}-\beta}\left[e^{-\beta\left(R\left(U_{n-1}\right)+c T_{n}\right)}-e^{-\lambda_{1}\left(R\left(U_{n-1}\right)+c T_{n}\right)}\right]\right.\right. \\
& \left.\left.+\frac{q \lambda_{2}}{\lambda_{2}-\beta}\left[e^{-\beta\left(R\left(U_{n-1}\right)+c T_{n}\right)}-e^{-\lambda_{2}\left(R\left(U_{n-1}\right)+c T_{n}\right)}\right]\right) \mid \Omega_{n-1}\right] \\
& =\frac{p \lambda_{1}}{\lambda_{1}-\beta} e^{-a U_{n-1}}\left[e^{-\beta R\left(U_{n-1}\right)} \gamma(a+c \beta)-e^{-\lambda_{1}\left(R\left(U_{n-1}\right)\right.} \gamma\left(a+c \lambda_{1}\right)\right] \\
& +\frac{q \lambda_{2}}{\lambda_{2}-\beta} e^{-a U_{n-1}}\left[e^{-\beta R\left(U_{n-1}\right)} \gamma(a+c \beta)-e^{-\lambda_{2}\left(R\left(U_{n-1}\right)\right.} \gamma\left(a+c \lambda_{2}\right)\right] .
\end{aligned}
$$

Therefore, by using (2.7) we get

$$
\begin{aligned}
\hat{H}(a, \beta)-e^{-\beta u} & =\sum_{n \geq 1} E\left[e^{-a U_{n}-\beta R\left(U_{n}\right)} I_{\{v>n\}}\right] \\
& =\sum_{n \geq 1} E\left[E\left(e^{-a U_{n}-\beta R\left(U_{n}\right)} I_{\{v>n\}} \mid \Omega_{n-1}\right)\right]
\end{aligned}
$$




$$
\begin{aligned}
& =\sum_{n \geq 1} E\left[I_{\{v>n-1\}} E\left(e^{-a U_{n}-\beta R\left(U_{n}\right)} I_{\left\{R\left(U_{n}\right) \geq 0\right\}} \mid \Omega_{n-1}\right)\right] \\
& =\frac{p \lambda_{1}}{\lambda_{1}-\beta}\left[\gamma(a+c \beta) \hat{H}(a, \beta)-\gamma\left(a+c \lambda_{1}\right) \hat{H}\left(a, \lambda_{1}\right)\right] \\
& +\frac{q \lambda_{2}}{\lambda_{2}-\beta}\left[\gamma(a+c \beta) \hat{H}(a, \beta)-\gamma\left(a+c \lambda_{2}\right) \hat{H}\left(a, \lambda_{2}\right)\right] .
\end{aligned}
$$

It is easy from (2.8) to get that

$$
\begin{gathered}
\hat{H}(a, \beta)=\frac{e^{-\beta u}-\frac{p \lambda_{1}}{\lambda_{1}-\beta} \gamma\left(a+c \lambda_{1}\right) \hat{H}\left(a, \lambda_{1}\right)-\frac{q \lambda_{2}}{\lambda_{2}-\beta} \gamma\left(a+c \lambda_{2}\right) \hat{H}\left(a, \lambda_{2}\right)}{1-\left(\frac{p \lambda_{1}}{\lambda_{1}-\beta}+\frac{q \lambda_{2}}{\lambda_{2}-\beta}\right) \gamma(a+c \beta)} \\
=\frac{\left(\lambda_{1}-\beta\right)\left(\lambda_{2}-\beta\right) e^{-\beta u}-p \lambda_{1}\left(\lambda_{2}-\beta\right) \gamma\left(a+c \lambda_{1}\right) \hat{H}\left(a, \lambda_{1}\right)-q \lambda_{2}\left(\lambda_{1}-\beta\right) \gamma\left(a+c \lambda_{2}\right) \hat{H}\left(a, \lambda_{2}\right)}{\left(\lambda_{1}-\beta\right)\left(\lambda_{2}-\beta\right)-\left[\lambda_{1} \lambda_{2}-\left(p \lambda_{1}+q \lambda_{2}\right) \beta\right] \gamma(a+c \beta)}
\end{gathered}
$$

Since $\beta_{1}(\alpha)$ and $\beta_{2}(\alpha)$ are two roots of Equation (2.2), and $\hat{H}(\alpha, \beta)$ is analytical, thus $\beta_{1}(a), \beta_{2}(a)$ satisfy the following equations:

$$
\begin{aligned}
& \left(\lambda_{1}-\beta_{1}(a)\right)\left(\lambda_{2}-\beta_{1}(a)\right) e^{-\beta_{1}(a) u}-p \lambda_{1}\left(\lambda_{2}-\beta_{1}(a)\right) \gamma\left(a+c \lambda_{1}\right) \hat{H}\left(a, \lambda_{1}\right) \\
& =q \lambda_{2}\left(\lambda_{1}-\beta_{1}(a)\right) \gamma\left(a+c \lambda_{2}\right) \hat{H}\left(a, \lambda_{2}\right), \\
& \left(\lambda_{1}-\beta_{2}(a)\right)\left(\lambda_{2}-\beta_{2}(a)\right) e^{-\beta_{2}(a) u}-p \lambda_{1}\left(\lambda_{2}-\beta_{2}(a)\right) \gamma\left(a+c \lambda_{1}\right) \hat{H}\left(a, \lambda_{1}\right) \\
& =q \lambda_{2}\left(\lambda_{1}-\beta_{2}(a)\right) \gamma\left(a+c \lambda_{2}\right) \hat{H}\left(a, \lambda_{2}\right) .
\end{aligned}
$$

Solving the above equations we obtain

$$
\begin{aligned}
& \gamma\left(a+c \lambda_{1}\right) \hat{H}\left(a, \lambda_{1}\right)=\frac{\left(\lambda_{1}-\beta_{1}(a)\right)\left(\lambda_{1}-\beta_{2}(a)\right)\left[\left(\lambda_{2}-\beta_{1}(a)\right) e^{-\beta_{1}(a) u}-\left(\lambda_{2}-\beta_{2}(a)\right) e^{-\beta_{2}(a) u}\right]}{p \lambda_{1}\left[\left(\lambda_{1}-\beta_{2}(a)\right)\left(\lambda_{2}-\beta_{1}(a)\right)-\left(\lambda_{1}-\beta_{1}(a)\right)\left(\lambda_{2}-\beta_{2}(a)\right)\right]}, \\
& \gamma\left(a+c \lambda_{2}\right) \hat{H}\left(a, \lambda_{2}\right)=\frac{\left(\lambda_{2}-\beta_{1}(a)\right)\left(\lambda_{2}-\beta_{2}(a)\right)\left[\left(\lambda_{1}-\beta_{2}(a)\right) e^{-\beta_{2}(a) u}-\left(\lambda_{1}-\beta_{1}(a)\right) e^{-\beta_{1}(a) u}\right]}{q \lambda_{2}\left[\left(\lambda_{1}-\beta_{2}(a)\right)\left(\lambda_{2}-\beta_{1}(a)\right)-\left(\lambda_{1}-\beta_{1}(a)\right)\left(\lambda_{2}-\beta_{2}(a)\right)\right]} .
\end{aligned}
$$

Thus

$$
\begin{aligned}
\hat{H}(a, \beta) & =\left[\left(\lambda_{1}-\beta\right)\left(\lambda_{2}-\beta\right)-\left[\lambda_{1} \lambda_{2}-\left(p \lambda_{1}+q \lambda_{2}\right) \beta\right] \gamma(a+c \beta)\right]^{-1} \\
& \times\left\{\left(\lambda_{1}-\beta\right)\left(\lambda_{2}-\beta\right) e^{-\beta u}-\left[\left(\lambda_{1}-\beta_{2}(a)\right)\left(\lambda_{2}-\beta_{1}(a)\right)-\left(\lambda_{1}-\beta_{1}(a)\right)\left(\lambda_{2}-\beta_{2}(a)\right)\right]^{-1}\right. \\
& \times\left[\left(\lambda_{2}-\beta\right)\left(\lambda_{1}-\beta_{1}(a)\right)\left(\lambda_{1}-\beta_{2}(a)\right)\left[\left(\lambda_{2}-\beta_{1}(a)\right) e^{-\beta_{1}(a) u}-\left(\lambda_{2}-\beta_{2}(a)\right) e^{-\beta_{2}(a) u}\right]\right. \\
& \left.\left.+\left(\lambda_{1}-\beta\right)\left(\lambda_{2}-\beta_{1}(a)\right)\left(\lambda_{2}-\beta_{2}(a)\right)\left[\left(\lambda_{1}-\beta_{2}(a)\right) e^{-\beta_{2}(a) u}-\left(\lambda_{1}-\beta_{1}(a)\right) e^{-\beta_{1}(a) u}\right]\right]\right\} .
\end{aligned}
$$


Let $\beta=0$, then

$$
\begin{aligned}
& \hat{H}(a, 0)=\frac{1}{1-\gamma(a)} \\
& \times\left\{1-\frac{\left(\lambda_{1}-\lambda_{2}\right)\left[\left(1-\frac{\beta_{1}(a)}{\lambda_{1}}\right)\left(1-\frac{\beta_{1}(a)}{\lambda_{2}}\right) \beta_{2}(a) e^{-\beta_{1}(a) u}-\left(1-\frac{\beta_{2}(a)}{\lambda_{1}}\right)\left(1-\frac{\beta_{2}(a)}{\lambda_{2}}\right) \beta_{1}(a) e^{-\beta_{2}(a) u}\right]}{\left(\lambda_{1}-\beta_{2}(a)\right)\left(\lambda_{2}-\beta_{1}(a)\right)-\left(\lambda_{1}-\beta_{1}(a)\right)\left(\lambda_{2}-\beta_{2}(a)\right)}\right\}
\end{aligned}
$$

By (2.6) and (2.10) we have

$$
\begin{aligned}
& a \int_{0}^{\infty} e^{-a t} \varphi(t, u) d t=\hat{H}(a, 0)(1-\gamma(a)) \\
& =1-\left(\lambda_{1}-\lambda_{2}\right) \frac{y_{1}(\alpha) \beta_{2}(\alpha) e^{-\beta_{1}(a) u}-y_{2}(a) \beta_{1}(\alpha) e^{-\beta_{2}(a) u}}{\left(\lambda_{1}-\beta_{2}(\alpha)\right)\left(\lambda_{2}-\beta_{1}(\alpha)\right)-\left(\lambda_{1}-\beta_{1}(\alpha)\right)\left(\lambda_{2}-\beta_{2}(\alpha)\right)},
\end{aligned}
$$

where $y_{1}(a)=\left(1-\frac{\beta_{1}(a)}{\lambda_{1}}\right)\left(1-\frac{\beta_{1}(a)}{\lambda_{2}}\right), y_{2}(\alpha)=\left(1-\frac{\beta_{2}(a)}{\lambda_{1}}\right)\left(1-\frac{\beta_{2}(a)}{\lambda_{2}}\right)$. Since $\psi(t, u)=1-\varphi(t, u)$, (2.1) follows immediately from (2.11).

Remark 2 Theorem 1 shows an exact numerical technique which requires merely numerical inversion of the Laplace transform of the ruin probability within finite time. Numerical methods of such an inversion could be found in Abate (1992), and we don't discuss it here. On the other hand, it is well known that the probabilities of ruin $\psi(t, u)$ and $\psi(u)$ can be identified with the virtual and limiting waiting time distributions, respectively, in a single server queue fed by a renewal process and having the service time distribution $B$. So, it is possible to prove Theorem 1 by virtue of related results from the theory of queues [see, for example, Prabhu $(1965,1980)]$.

\section{Two COROLlaries}

As one application of Theorem 1, we can get the following two corollaries. The first Corollary is about the expression for Laplace transform of the ruin probability within finite time when the claim amount distribution is an exponential, which was obtained by Malinovskii (1998). The second Corollary is concerned with a result about ultimate ruin probability in the classical risk model which was proved by Gerber (1979).

Corollary 1 [Malinovskii (1988)] Let the sizes of claims $\left\{Y_{i}, i \geq 1\right\}$ and the interclaims $\left\{T_{i}, i \geq 1\right\}$ be iid and mutually independent. Assume that $Y_{1} \sim$ Exponential $(\lambda), \lambda>0$, and $\gamma(\alpha)=\int_{0}^{\infty} e^{-a u} P_{T}(d u)$ is the Laplace transform of $\mathbf{T}_{1}$. Then

$$
a \int_{0}^{\infty} e^{-a t} \varphi(t, u) d t=1-y(\alpha) \exp \{-u \lambda(1-y(a))\}, a>0
$$


where $y(a)$ is the unique root in $(0,1)$ of Equation

$$
y=\gamma(a+c \lambda(1-y)), a>0
$$

Proof Taking $p=1, \lambda_{1}=\lambda$ in (2.2) of Theorem 1 yields

$$
\left(\lambda_{2}-\beta\right)(\lambda-\beta-\lambda \gamma(\alpha+c \beta))=0, \alpha>0 .
$$

Therefore we get that $\beta_{2}(\alpha)=\lambda_{2}$, and $\beta_{1}(\alpha)$ is the unique root of Equation

$$
\lambda-\beta-\lambda \gamma(a+c \beta)=0, a>0 .
$$

Moreover, $y_{1}(\alpha)=\left(1-\frac{\beta_{1}(\alpha)}{\lambda}\right)\left(1-\frac{\beta_{1}(a)}{\lambda_{2}}\right), y_{2}(\alpha)=0$, and the right side of $(2.1)$ is $\left(1-\frac{\beta_{1}(\alpha)}{\lambda}\right)$ $e^{-\beta_{1}(a) u}$. Put $y(a)=1-\frac{\beta_{1}(a)}{\lambda}$. Then

$$
\left(1-\frac{\beta_{1}(a)}{\lambda}\right) e^{-\beta_{1}(\alpha) u}=y(\alpha) \exp \{-u \lambda(1-y(\alpha))\} .
$$

Again by (3.4) we see that $y(a)$ is the unique root of the Equation $(3.2)$ in $(0,1)$. Finally, (3.1) follows immediately from $(2.1)$ by $\psi(t, u)=1-\varphi(t, u)$.

Corollary 2 [Gerber (1979)] For the classical risk model, in the case of exponential claim amounts, the ultimate ruin probability is an exponential function of the initial surplus measured in mean claim amounts. In other words, if the interclaims $\left\{T_{i}, i \geq 1\right\} \sim \operatorname{Exponential}(\mu), \mu>0$, the sizes of claims $\left\{Y_{i}, i \geq 1\right\} \sim$ Exponential $(\lambda), \lambda>0$. Then $\psi(u)=\frac{1}{1+\Lambda} \exp \left\{-\frac{\Lambda}{1+\Lambda} \frac{u}{E\left(Y_{1}\right)}\right\}$, where $\Lambda=\frac{c}{\mu E Y_{1}}-1$ $=\frac{c \lambda}{\mu}-1$.

Before we give the proof of this Corollary, we need the following Lemma, whose proof is very easy by induction, and so is omitted.

Lemma 1 When $0<x \leq \frac{1}{4}$, for any nonnegative integer $n$, the following equality always holds:

$$
\sum_{k=0}^{\infty} \frac{(n+2 k) !}{k !(n+k+1) !} x^{k}=\frac{1}{(n+1)}\left(\frac{1+\sqrt{1-4 x}}{2}\right)^{-(n+1)} .
$$

Remark 3 When $0<p<1,0<q<1, p+q=1$, for any nonnegative interger $n$,

$$
\sum_{k=0}^{\infty} \frac{(n+1)(n+2 k) !}{k !(n+k+1) !} p^{k} q^{n+k+1}=\left\{\begin{array}{cl}
1, & 0<p \leq \frac{1}{2}, \\
\left(\frac{q}{p}\right)^{n+1}, & \frac{1}{2}<p<1 .
\end{array}\right.
$$

Proof Evidently, $0<p q \leq \frac{1}{4}$. Therefore by Lemma 1 we see that

$$
\sum_{k=0}^{\infty} \frac{(n+2 k) !}{k !(n+k+1) !}(p q)^{k}=\frac{1}{n+1}\left(\frac{1+\sqrt{1-4 p q}}{2}\right)^{-(n+1)} .
$$


Consequently,

$$
\sum_{k=0}^{\infty} \frac{(n+1)(n+2 k) !}{k !(n+k+1) !} p^{k} q^{n+k+1}=q^{n+1}\left(\frac{1+|1-2 p|}{2}\right)^{-(n+1)}=\left\{\begin{array}{cl}
1, & 0<p \leq \frac{1}{2} \\
\left(\frac{q}{p}\right)^{n+1}, & \frac{1}{2}<p<1
\end{array}\right.
$$

The following (3.7) comes immediately from the above (3.6).

Remark 4 Let $p=\frac{1}{2+\Lambda}, q=\frac{1+\Lambda}{2+\Lambda}$, where $\Lambda$ is the relative safety loading, $\Lambda>0$. Then for any nonnegative integer $n$ we have

$$
\sum_{k=0}^{\infty} \frac{(n+1)(n+2 k) !}{k !(n+k+1) !}\left(\frac{1}{2+\Lambda}\right)^{k}\left(\frac{1+\Lambda}{2+\Lambda}\right)^{n+k+1}=1
$$

The Proof of Corollary 2 Since $\left\{T_{i}, i \geq 1\right\} \sim E(\mu), \mu>0, \gamma(a)=\frac{\mu}{a+\mu}$, by Corollary 1 we have $y(a)=\gamma(a+c \lambda(1-y(a)))=\frac{\mu}{a+c \lambda(1-y(a))+\mu}$, i.e.,

$$
c \lambda y^{2}(a)-(a+\mu+c \lambda) y(a)+\mu=0 \text {. }
$$

Again by Corollary 1 we get

$$
y(a)=4 \mu\left(\sqrt{a+(\sqrt{\mu}+\sqrt{c \lambda})^{2}}+\sqrt{a+(\sqrt{\mu}+\sqrt{c \lambda})^{2}}\right)^{-2} .
$$

Put $x=(\sqrt{\mu}+\sqrt{c \lambda})^{2}, y=(\sqrt{\mu}-\sqrt{c \lambda})^{2}$, then

$$
y(a)=\sqrt{\frac{\mu}{c \lambda}}(x-y)(\sqrt{a+x}+\sqrt{a+y})^{-2} .
$$

Thus $y(a)$ is the Laplace transform of $\sqrt{\frac{\mu}{c \lambda}} e^{-(\mu+c \lambda) t} t^{-1} I_{1}(2 \sqrt{\mu c \lambda t})$, where $\mathrm{I}_{1}(z)=$ $\sum_{k=0}^{\infty} \frac{1}{k ! \Gamma(k+2)}\left(\frac{z}{2}\right)^{2 k+1}$ is the first kind of Bessel function (see Feller (1971)). On the other hand, $a \int_{0}^{\infty} e^{-a t} \varphi(t, u) d t=1-y(a) \exp \left\{-u \lambda(1-y(a)\}, a>0\right.$, and $a \int_{0}^{\infty} e^{-a t}$ $\varphi(t, u) d t=1-E\left(e^{-a t}\right)$. Hence

$$
\begin{aligned}
E\left(e^{-a \tau}\right) & =e^{-u \lambda} \sum_{n=0}^{\infty} \frac{(u \lambda)^{n}}{n !} y^{n+1}(\alpha) \\
& =e^{-u \lambda} \sum_{n=0}^{\infty} \frac{(u \lambda)^{n}}{n !}\left(\sqrt{\frac{\mu}{c \lambda}}\right)^{n+1}\left((x-y)(\sqrt{a+x}+\sqrt{a+y})^{-2}\right)^{n+1} .
\end{aligned}
$$

The (3.10) is the Laplace transform of $e^{-u \lambda} \sum_{n=0}^{\infty} \frac{\left(u \lambda \sqrt{\frac{\mu}{c \lambda}}\right)^{n}}{n ! t} \sqrt{\frac{\mu}{c \lambda}}(n+1) e^{-(\mu+c \lambda) t} \mathrm{I}_{n+1}(2 \sqrt{\mu c \lambda t})$, where $\mathrm{I}_{n}(z)=\sum_{k=0}^{\infty} \frac{1}{k ! \Gamma(n+k+1)}\left(\frac{z}{2}\right)^{n+2 k}$ (see Feller (1971)). Hence 


$$
\begin{aligned}
\psi(t, u) & =P(\tau \leq t) \\
& =\int_{0}^{t} e^{-u \lambda} \sum_{n=0}^{\infty} \frac{(u \lambda \sqrt{\mu / c \lambda})^{n}}{n ! s} \sqrt{\frac{\mu}{c \lambda}}(n+1) e^{-(\mu+c \lambda) s} I_{n+1}(2 \sqrt{\mu c \lambda} s) d s \\
& =\frac{\mu}{c \lambda} e^{-u \lambda} \sum_{n=0}^{\infty} \frac{\left(\frac{u \mu}{c}\right)^{n}}{n !}\left(\frac{\mu}{c \lambda}\right)^{-\frac{n+1}{2}} \int_{0}^{t} e^{-(\mu+c \lambda) s} \frac{n+1}{s} I_{n+1}(2 \sqrt{\mu c \lambda} s) d s .
\end{aligned}
$$

Thus, letting $t \rightarrow \infty$ and using Remark 4 gives

$$
\begin{aligned}
& \psi(u)=P(\tau<\infty) \\
& =\frac{\mu}{c \lambda} e^{-u \lambda} \sum_{n=0}^{\infty} \frac{\left(\frac{u \mu}{c}\right)^{n}}{n !}\left(\frac{\mu}{c \lambda}\right)^{-\frac{n+1}{2}} \int_{0}^{\infty} e^{-(\mu+c \lambda) s} \frac{n+1}{s} I_{n+1}(2 \sqrt{\mu c \lambda} s) d s \\
& =\frac{\mu}{c \lambda} e^{-u \lambda} \sum_{n=0}^{\infty} \frac{\left(\frac{u \mu}{c}\right)^{n}}{n !}\left(\frac{\mu}{c \lambda}\right)^{-\frac{n+1}{2}} \int_{0}^{\infty} e^{-(\mu+c \lambda) s} \frac{n+1}{s} \sum_{k=0}^{\infty} \frac{1}{k ! \Gamma(n+k+2)}(\sqrt{\mu c \lambda} s)^{n+2 k+1} d s \\
& =\frac{\mu}{c \lambda} e^{-u \lambda} \sum_{n=0}^{\infty} \frac{\left(\frac{u \mu}{c}\right)^{n}}{n !}\left(\frac{\mu}{c \lambda}\right)^{-\frac{n+1}{2}}(n+1) \sum_{k=0}^{\infty} \frac{\Gamma(n+2 k+1)}{k ! \Gamma(n+k+2)} \frac{(\sqrt{\mu c \lambda})^{n+2 k+1}}{(\mu+c \lambda)^{n+2 k+1}} \\
& =\frac{\mu}{c \lambda} e^{-u \lambda} \sum_{n=0}^{\infty} \frac{n+1}{n !}\left(\frac{u \mu}{c}\right)^{n} \sum_{k=0}^{\infty} \frac{\Gamma(n+2 k+1)}{k ! \Gamma(n+k+2)}\left(\frac{c \lambda}{\mu}\right)^{n+k+1}\left(1+\frac{c \lambda}{\mu}\right)^{-(n+2 k+1)} \\
& =\frac{1}{1+\Lambda} e^{-u \lambda} \sum_{n=0}^{\infty} \frac{1}{n !}\left(\frac{u \lambda}{1+\Lambda}\right)^{n}(n+1) \sum_{k=0}^{\infty} \frac{(n+2 k) !}{k !(n+k+1) !}\left(\frac{1+\Lambda}{2+\Lambda}\right)^{n+k+1}\left(\frac{1}{2+\Lambda}\right)^{k} \\
& =\frac{1}{1+\Lambda} e^{-u \lambda} e^{\frac{\lambda u}{1+\Lambda}} \\
& =\frac{1}{1+\Lambda} \exp \left\{-\frac{\Lambda}{1+\Lambda} \lambda u\right\} \\
& =\frac{1}{1+\Lambda} \exp \left\{-\frac{\Lambda}{1+\Lambda} \frac{u}{E(Y)}\right\} .
\end{aligned}
$$

\section{REFERENCES}

Abate J. and WhITT W. (1992). Fourier series method for inverting transforms of probability distributions, Queuing Systems 10, 5-88.

Dickson, D.C.M. (1998). On a class of renewal risk processes. NAAJ, 2, No. 2, 60-73.

FELLer, W. (1971). An Introduction to Probability Theory and its Applications, 2nd ed., Vol. 2, Wiley, New York.

GERBER, H.U. (1979). An Introduction to Mathematical Risk Theory, S.S. Huebner Foundation Monograph Series 8, Philadelphia, 114-116.

GRANDELl, J. (1991). Aspects of Risk Theory, Springer, New York. 
MALINOVSKII, V.K. (1998). Non-poissonian claims' arrivals and calculation of the probability of ruin. Insurance: Mathematics \& Economics, Vol. 22, 123-138.

Prabnu, N.U. (1965). Queues and Inventories. J. Wiley \& Sons, New York.

Prabnu, N.U. (1980). Stochastic Storage Processes. Queues, Insurance Risk, and Dams. Springer, New York.

Sparre Andersen, E. (1957). On the Collective Theory of Risk in the Case of Contagion between the Claims, Transactions of the XV International Congress of Actuaries 2: 212-219.

\author{
WANG RONG-MING \\ Department of Statistics \\ East China Normal University \\ 3663 Zhongshan Road (northern) \\ Shanghai 200062 \\ P.R. China
}

